\title{
Glycogen hepatopathy in children with poorly controlled type 1 diabetes
}

\author{
Hanh T D Vo, Genna W Klein, Anthony Loizides \\ Ping Zhou, Qiang Liu, Debra H Pan
}

\begin{abstract}
Introduction: Glycogen hepatopathy has been described in children with poorly controlled type 1 diabetes mellitus (T1DM). Diabetic patients with hepatomegaly and abnormal liver enzymes have often been assumed to have nonalcoholic steatohepatitis rather than glycogen hepatopathy. It is important to recognize and distinguish glycogen hepatopathy from nonalcoholic steatohepatitis as it impacts the management and long-term prognosis. Case Report: We describe four cases of children with poorly controlled type 1 diabetes mellitus who developed glycogen hepatopathy, and review the available literature. Conclusion: Pediatricians should raise awareness of this potentially reversible liver complication in children with poorly controlled T1DM. It has
\end{abstract}

Hanh T. D. V ${ }^{1}$, Genna W. Klein ${ }^{2}$, Anthony Loizides ${ }^{3}$, Ping Zhou ${ }^{2}$, Qiang Liu ${ }^{4}$, Debra H. Pan ${ }^{3}$

Affiliations: Department of Pediatrics, Bronx-Lebanon Hospital Center, Bronx, NY, United States; ${ }^{2}$ Division of Pediatric Endocrinology, Department of Pediatrics, The Children's Hospital at Montefiore, Bronx, NY, United States; ${ }^{3}$ Division of Pediatric Gastroenterology, Department of Pediatrics, The Children's Hospital at Montefiore, Bronx, NY, United States; ${ }^{4}$ Department of Pathology, The Children's Hospital at Montefiore, Bronx, NY, United States.

Corresponding Author: Debra H. Pan, Division of Pediatric Gastroenterology, Department of Pediatrics, The Children's Hospital at Montefiore, Bronx, NY 10467, USA; Ph: 1(718) 741 2332; Fax: 1(718) 515 5426;

Email: dpan@montefiore.org

Received: 04 March 2011

Accepted: 16 May 2011

Published: 01 September 2011 been suggested that diabetic patients with hepatomegaly and elevated liver enzymes be given a trial of improved glycemic control prior to more invasive investigation.

Keywords: Children, Hepatomegaly, Diabetes, Glycogen hepatopathy

$$
* * * * * * * * *
$$

Vo HTD, Klein GW, Loizides A, Zhou P, Liu Q, Pan DH. Glycogen hepatopathy in children with poorly controlled type 1 diabetes. International Journal of Case Reports and Images 2011;2(9):1-4.

$$
* * * * * * * * *
$$

doi:10.5348/ijcri-2011-09-51-CS-1

\section{INTRODUCTION}

Type 1 diabetes (T1DM) is a common pediatric endocrine condition. Although glycogen hepatopathy (GH) has been described as one of the manifestations of poorly controlled T1DM [1, 2], diabetic patients with hepatomegaly and abnormal liver enzymes have often been assumed to have fatty liver disease or nonalcoholic steatohepatitis (NASH) rather than GH. It has been reported that GH occurs more often in children or adolescents with T1DM as a result of hepatic response to poor glycemic control, whereas NASH is more likely seen in obese patients with type 2 diabetes $[1,3]$. GH also has distinct histologic features, and is reversible with good glycemic control [3], whereas NASH has been reported to progress to fibrosis in up to $50 \%$ and cirrhosis in up to $15 \%$ of adult patients [4]. It is important to recognize and distinguish GH from NASH as it impacts the management and long-term prognosis. Here we report four diabetic children who developed glycogen 
hepatopathy. We aim to raise awareness of this potentially reversible liver complication in T1DM.

\section{CASE REPORT}

Case 1: A 12-year-old African American female was diagnosed with T1DM at six years of age. She first presented in the pediatric gastroenterology (GI) clinic at 10 years of age for evaluation of abdominal pain. Past medical history was significant for poor glycemic control with multiple admissions for diabetic ketoacidosis (DKA). Physical examination revealed weight and height between the $50^{\text {th }}$ and $75^{\text {th }}$ percentiles. Her hemoglobin A1c (HbA1c) ranged from 10 to $12 \%$. Her first liver ultrasound showed normal size and echotexture. Repeat abdominal ultrasound six months later revealed an enlarged liver measuring 19 $\mathrm{cm}$ with a heterogeneous texture. Her liver tests showed aspartate aminotransferase (AST) - $33 \mathrm{U} / \mathrm{L}$, alanine aminotransferase (ALT) - $64 \mathrm{U} / \mathrm{L}$. Two months later, she was admitted for DKA, and her liver enzymes worsened (AST - 983 U/L, ALT - 494 U/L). A repeat liver ultrasound showed an enlarged liver with a hyperechoic echotexture. A liver biopsy was subsequently performed which revealed findings consistent with diffuse glycogen-rich cytoplasm and glycogenated nuclei of hepatocytes; there was no steatosis, fibrosis or inflammation. She was placed in a foster home and attended an intensive diabetic control program. Her HbA1c decreased from 12\% to $10 \%$; her hepatomegaly resolved and liver tests improved after four weeks of proper glycemic control. She maintained her HbA1c between 8 - 9\% thereafter with no recurrence of hepatomegaly or transaminase elevation.

Case 2: A 14-year-old Hispanic male was admitted for evaluation of lower leg edema and massive hepatomegaly. He was diagnosed with T1DM at three years of age. His glycemia had been poorly controlled resulting in several episodes of DKA. Physical exam demonstrated an enlarged liver palpable to the umbilicus. His weight was between $25^{\text {th }}$ and $50^{\text {th }}$ percentiles and his height was below $5^{\text {th }}$ percentile. He was in Tanner 1 for pubic hair and Tanner 3 for testicles. His HbA1c ranged from 9 to $12 \%$. His liver tests showed AST - $878 \mathrm{U} / \mathrm{L}$, ALT - $751 \mathrm{U} / \mathrm{L}$. The liver ultrasound showed markedly enlarged liver measuring $26 \mathrm{~cm}$ in span. After evaluation for chronic liver disease was negative, a liver biopsy was performed. The pathology reported glycogen-rich cytoplasm and glycogenated nuclei of hepatocytes with no steatosis, inflammation or fibrosis (Figures 1 and 2). $\mathrm{He}$ underwent strict diabetic control, his liver enzymes returned to normal within 4 weeks (AST - 24 U/L, ALT 26 - U/L, HbA1c - 8.2\%). Unfortunately, due to multiple social circumstances, he could not maintain good glycemic control. He continues to have hepatomegaly, a high HbA1c of $12 \%$ and mild transaminase elevation.

Case 3: An 18-year-old Hispanic female was diagnosed with T1DM at three years of age. Her



Figure 1: Hematoxylin and eosin stain of needle liver biopsy showed hepatocytes diffusely swollen and enlarged, with rarified cytoplasm and centrally placed nuclei. The cells were arrayed in a mosaic pattern throughout the lobules.

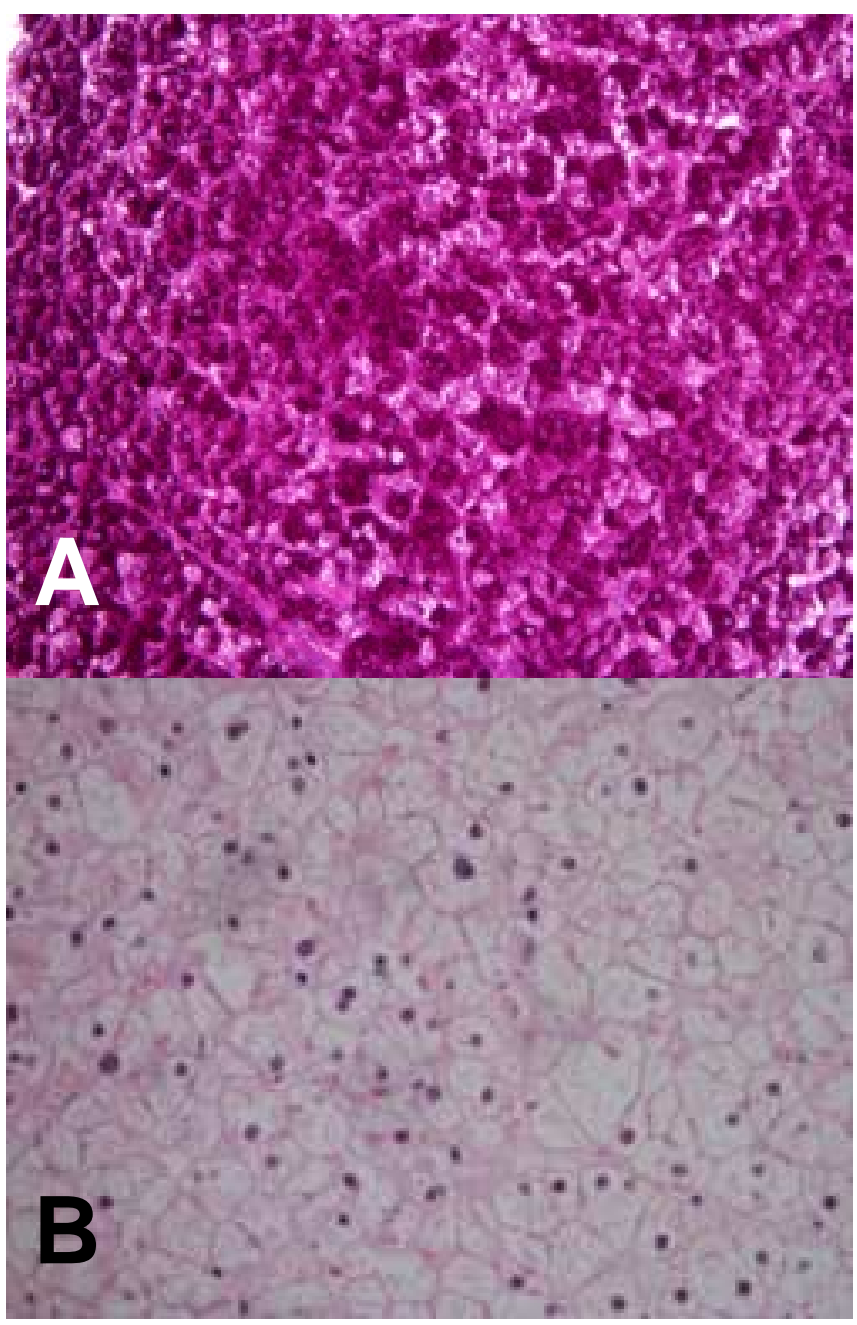

Figure 2: Periodic Acid-Schiff stain showed A) strong cytoplasm positivity, which B) disappeared upon pretreatment with diastase (PASD stain), consistent with glycogen accumulation. 
diabetes was poorly controlled resulting in more than 20 episodes of DKA. Her HbA1c ranged from 13 to $17 \%$. She first presented to the pediatric GI clinic at 16 years of age for evaluation of hepatomegaly and markedly elevated transaminases. Her weight was between $25^{\text {th }}$ and $50^{\text {th }}$ percentiles, and her height was at $5^{\text {th }}$ percentile. She was in Tanner 4 for breast development and pubic hair. An abdominal CT scan done at 14 years of age demonstrated hepatomegaly with a $20 \mathrm{~cm}$ liver span. Her liver tests revealed AST $3915 \mathrm{U} / \mathrm{L}$ and ALT - $1037 \mathrm{U} / \mathrm{L}$, which markedly decreased three days later (AST - $166 \mathrm{U} / \mathrm{L}$, ALT - 359 $\mathrm{U} / \mathrm{L}$ ), suggesting an acute insult to the liver. The evaluation for viral hepatitis, autoimmune hepatitis, celiac disease and metabolic liver diseases was negative. Liver biopsy was performed, which revealed findings consistent with diffuse hepatocellular glycogen accumulation. There was no steatosis, fibrosis or inflammation. She was admitted eight months after the liver biopsy for another episode of DKA. She was then enrolled in an intensive diabetic control program but could not maintain euglycemic control (HbA1c - 16 $\%)$; her repeat ultrasound showed markedly enlarged liver measuring $27 \mathrm{~cm}$ in span; her transaminases remained mildly elevated (AST - 75 U/L, ALT - 49 $\mathrm{U} / \mathrm{L}$ ).

Case 4: A 12-year-old Hispanic female was diagnosed with T1DM at nine years of age. She had poor glycemic control with multiple admissions for DKA. Her HbA1c ranged from 10 to $13 \%$. At 10 years of age, the pediatric GI service was consulted for evaluation of elevated liver transaminases (AST 528 U/L, ALT 216 U/L) during her episode of DKA. Her weight was at the $50^{\text {th }}$ percentile, and her height was between the $75^{\text {th }}$ and $90^{\text {th }}$ percentiles. An abdominal ultrasound showed normal size and echotexture of the liver. Her liver enzymes improved prior to discharge. Two years later, she returned to the GI clinic for evaluation of significant abdominal distention and elevated liver enzymes. A repeat abdominal ultrasound revealed massive hepatomegaly and mixed echogenicity with liver span measuring $20 \mathrm{~cm}$. Her liver tests showed AST - $153 \mathrm{U} / \mathrm{L}$, ALT - $133 \mathrm{U} / \mathrm{L}$. The evaluation for viral hepatitis, autoimmune hepatitis, celiac disease, and metabolic liver diseases was negative. She did not return for follow-up. The liver biopsy was not performed, but GH was strongly suspected based on her poor glycemic control.

\section{DISCUSSION}

Since Mauriac first described a syndrome of hepatomegaly with abnormal liver enzymes, growth retardation, delayed puberty, cushingoid features, and hypercholesterolemia in poorly controlled T1DM [5], a growing number of cases have been described detailing glycogen-induced hepatomegaly in the setting of poorly controlled diabetes [1-3]. Most patients presented with abdominal pain and distention. All patients had markedly increased liver transaminases at one point in time suggesting some form of acute hepatitis. These elevations were not associated with DKA in cases 2 and 3 . In cases 1 and 4, elevated transaminases occurred during DKA episodes, which might be caused by severe metabolic acidosis. Those high values quickly declined to normal or near normal within days or weeks. This phenomenon may represent that glycogen-rich hepatocytes are more susceptible to any kind of acute insult such as metabolic acidosis or viral infection. All patients had a long-standing history of poorly controlled T1DM, but none presented with classic features of Mauriac syndrome, suggesting that GH could be an early sign and euglycemic control might be the key to prevent full picture of Mauriac syndrome.

Diabetic patients with hepatomegaly and abnormal liver enzymes have often been assumed to have NASH rather than GH. A liver ultrasound is not helpful in distinguishing NASH from GH. Consequently, the condition often goes under-recognized with no further evaluation. GH is a liver condition with minor chance or no risk of progression to fibrosis and is reversible with euglycemic control $[1,3]$. In contrast, pediatric NASH has been reported to be associated with cirrhosis [6-10], and adolescents with end-stage liver disease caused by NASH have undergone liver transplantation [10, 11]. Thus, it is important to distinguish GH from NASH as its management and prognosis are distinct from those of NASH.

Liver biopsy is helpful to confirm the diagnosis of GH. Histologic features in GH are characterized by large, swollen, glycogen-laden hepatocytes and glycogenated nuclei without significant fatty change, inflammation, lobular spotty necrosis or fibrosis [2]. This was evident in our three biopsy cases. Steatohepatitis is defined as the combination of steatosis, lobular inflammation, acidophilic bodies, and pericellular fibrosis. The differential diagnosis of GH includes NASH and glycogen storage diseases. However, the diagnosis of T1DM-associated GH can be considered based on a history of poorly controlled T1DM and response to euglycemic control. Other features of Mauriac syndrome, though not necessary, can, if present, assist with the diagnosis.

The pathogenesis of $\mathrm{GH}$ has been previously described $[1,2]$. T1DM-associated GH results from excess accumulation of glycogen in hepatocytes, frequently seen in unstable diabetic patients, where hyperglycemia is treated with supra-physiologic doses of insulin. Blood glucose passively enters the hepatocytes in which glycogen synthesis is promoted by high cytoplasmic glucose concentration reliant on the presence of insulin. Glycogen is then trapped within the hepatocytes as a result of a vicious cycle of hyperglycemia and insulin treatment. This contrasts to NASH, where lipid accumulation in the liver develops as a result of chronic hyperinsulinemia and hepatocellular insulin resistance $[7,12,13]$. 


\section{CONCLUSION}

In conclusion, pediatricians should have a higher level of awareness of the possible development of GH in children with poorly controlled T1DM, and should perform a focused examination of the abdomen to assess for hepatomegaly. Although liver biopsy does confirm the diagnosis of $\mathrm{GH}$, it may not be needed in the setting of massive hepatomegaly and poorly controlled T1DM. It has been suggested that diabetic patients with hepatomegaly and elevated liver enzymes be given a trial of improved glycemic control prior to more invasive investigations [1]. As diabetic children may have unfavorable social circumstances that could aggravate their glycemic control, a multidisciplinary approach in diabetic care should be emphasized to prevent diabetes related liver disease.

$$
* * * * * * * * *
$$

\section{Author Contributions}

Hanh T. D. Vo - Conception and design, Acquisition of data, Analysis and interpretation of data, Drafting the article, Critical revision of the article, Final approval of the version to be published.

Genna W. Klein - Analysis and interpretation of data, Critical revision of the article, Final approval of the version to be published.

Anthony Loizides - Analysis and interpretation of data, Critical revision of the article, Final approval of the version to be published.

Ping Zhou - Analysis and interpretation of data, Critical revision of the article, Final approval of the version to be published.

Qiang Liu - Acquisition of data, Analysis and interpretation of data, Critical revision of the article, Final approval of the version to be published.

Debra H. Pan - Conception and design, Acquisition of data, Analysis and interpretation of data, Drafting the article, Critical revision of the article, Final approval of the version to be published.

\section{Guarantor}

The corresponding author is the guarantor of submission.

\section{Conflict of Interest}

Authors declare no conflict of interest.

\section{Copyright}

(C) Hanh T. D. Vo et al. 2011; This article is distributed under the terms of Creative Commons attribution 3.0 License which permits unrestricted use, distribution and reproduction in any means provided the original authors and original publisher are properly credited. (Please see www.ijcasereportsandimages.com /copyright-policy.php for more information.)

\section{REFERENCES}

1. Munns CF, McCrossin RB, Thomsett MJ, Batch J. Hepatic glycogenosis: reversible hepatomegaly in type 1 diabetes. $\mathrm{J}$ Paediatr Child Health. 2000;36:449-52.

2. Torbenson M, Chen YY, Brunt E, Cummings OW, Gottfried M, Jakate S, et al. Glycogenic hepatopathy: an underrecognized hepatic complication of diabetes mellitus. Am J Surg Pathol. 2006;30:508-13.

3. Chatila R, West AB. Hepatomegaly and abnormal liver tests due to glycogenosis in adults with diabetes. Medicine (Baltimore). 1996;75:327-33.

4. Sheth SG, Gordon FD, Chopra S. Nonalcoholic steatohepatitis. Ann Intern Med. 1997;126:137-45.

5. Mauriac P. Gros ventre, hépatomégalie, troubles de la croissance chez les enfants diabetiques: traités depuis plusieurs années par l'insuline. Gaz Hebl Sci Med Bordeaux. 1930:402-10.

6. Schwimmer JB, Deutsch R, Rauch JB, Behling C, Newbury R, Lavine JE. Obesity, insulin resistance, and other clinicopathological correlates of pediatric nonalcoholic fatty liver disease. $\mathrm{J}$ Pediatr. 2003;143:500-5.

7. Rashid M, Roberts EA. Nonalcoholic steatohepatitis in children. $\mathrm{J}$ Pediatr Gastroenterol Nutr. 2000;30:48-53.

8. Kinugasa A, Tsunamoto K, Furukawa N, Sawada T, Kusunoki T, Shimada N. Fatty liver and its fibrous changes found in simple obesity of children. J Pediatr Gastroenterol Nutr. 1984;3:408-14.

9. Willner IR, Waters B, Patil SR, Reuben A, Morelli J, Riely CA. Ninety patients with nonalcoholic steatohepatitis: insulin resistance, familial tendency, and severity of disease. Am J Gastroenterol. 2001;96:2957-61.

10. Molleston JP, White F, Teckman J, Fitzgerald JF. Obese children with steatohepatitis can develop cirrhosis in childhood. Am J Gastroenterol. 2002;97:2460-2.

11. Jonas MM, Krawczuk LE, Kim HB, Lillehei C, PerezAtayde A. Rapid recurrence of nonalcoholic fatty liver disease after transplantation in a child with hypopituitarism and hepatopulmonary syndrome. Liver Transpl. 2005;11:108-10.

12. Loomba R, Sirlin CB, Schwimmer JB, Lavine JE. Advances in pediatric nonalcoholic fatty liver disease. Hepatology. 2009;50:1282-93.

13. Brunt EM. Nonalcoholic steatohepatitis. Semin Liver Dis. 2004;24:3-20. 